\title{
Faust : Walpurgisnacht
}

Faust, Walpurgisnacht

Faust: Walpurgisnacht

\section{Denise Blondeau}

\section{OpenEdition \\ Journals}

Édition électronique

URL : https://journals.openedition.org/ceg/12098

DOI : $10.4000 /$ ceg. 12098

ISSN : 2605-8359

\section{Éditeur}

Presses Universitaires de Provence

\section{Édition imprimée}

Date de publication : 1 avril 2012

Pagination : 251-249

ISBN : 0751-4239

ISSN : 0751-4239

\section{Référence électronique}

Denise Blondeau, « Faust : Walpurgisnacht », Cahiers d'Études Germaniques [En ligne], 62 | 2012, mis en ligne le 12 octobre 2020, consulté le 07 juin 2021. URL : http://journals.openedition.org/ceg/12098 ; DOI : https://doi.org/10.4000/ceg.12098 


\section{Faust : Walpurgisnacht \\ Denise BLONDEAU \\ Reims / Paris}

Captée par l'imaginaire collectif, puis par le discours littéraire aux époques agitées, travaillées par des germinations souterraines d'abord confuses, la figure de Faust est toujours synonyme de transgression et emblème de modernité ${ }^{1}$. Il est alors logique que le personnage et le thème soient porteurs des contradictions et paradoxes qui affectent ces époques où un ordre symbolique normatif se fissure, où un changement d'épistémè bouleverse les consciences et les pensées. Le discours littéraire sur Faust évolue : le conflit fondamental entre un ordre et le personnage transgressif reste, mais la volonté édificatrice des récits s'estompe, la force des dogmes s'émousse, les actes et les postulations de Faust sont examinés par rapport à des références nouvelles. Le personnage gagne en épaisseur, en complexité et en opacité. Sa modernité n'est plus condamnée, comme hérésie ou comme démesure, mais perçue comme ambiguïté, voire comme malheur et comme souffrance. Cette mise en question des discours anciens entraîne une dramatisation du personnage et de l'histoire, par les scènes ambulantes et théâtres de marionnettes, avec le drame sérieux de Christopher Marlowe et la "tragédie" de Goethe. On sait avec quelle circonspection Goethe use de la terminologie "tragédie", "tragique". La réflexion anthropologique, conduite à travers les thématiques de la connaissance et de l'action, du mal et de la culpabilité, de la responsabilité et de la liberté, de l'ordre social et de sa transgression, de la justice humaine, des relations entre les sexes... impose un théâtre tragique moderne. Dès lors, que faire, dans cette tragédie, dont la rédaction est commencée dans le dernier tiers $d u X V_{I I I}{ }^{\mathrm{e}}$ siècle et achevée pendant le premier tiers du $\mathrm{XIX}^{\mathrm{e}}$, du ballast obligatoire que sont les sorcières et le diable ? Il ne faut pas oublier ce qui est peut-être un paradoxe, ou une "polarité" au sens goethéen : les grandes scènes de sorcellerie de Faust ont été composées en Italie et après le retour d'Italie, dans cette période du tournant du siècle que la critique subsume volontiers sous la référence

1 On peut se reporter à propos de cette "modernité" du thème à Béatrice DUMICHE / Denise BlondeAu (éd.), Faust, modernisation d'un modèle, Paris, L'Harmattan, 2005. 
temporelle "um 1800", où la réflexion anthropologique et esthétique trouve une intensité encore jamais atteinte. L'élaboration d'une esthétique idéaliste et les percées décisives du poète dans les sciences de la nature, puis les sciences des vivants et enfin du vivant, semblent alors l'éloigner des fantômes nordiques. Un Faust moderne doit donc revisiter les figures du diable et des sorcières.

On connaît les difficultés rencontrées par le dramaturge pour élaborer la figure du diable et camper celle de Méphisto. Méphisto lui-même pose le problème : ni l'étudiant, dans la scène Studierzimmer, ni les étudiants de la scène Auerbachs Keller, ni même la sorcière de Hexenküche n'ont reconnu le diable, et face à cette dernière Méphisto déplore :

Auch die Kultur, die alle Welt beleckt, / Hat auf den Teufel sich erstreckt ; / Das nordische Phantom ist nun nicht mehr zu schauen ; [...] / Er [der Junker Satan] ist schon lang' ins Fabelbuch geschrieben ${ }^{2}$.

La même injonction de modernité est appliquée aux sorcières et leur fonction maléfique. La vieille sorcière brocanteuse de la Nuit de Walpurgis propose tout un bric à brac d'instruments de mort et cite les actes criminels répertoriés par les tribunaux ou les théâtres divers :

Ihr Herren, [...] / Aufmerksam blickt nach meinen Waren, / [...] Kein Dolch ist hier, von dem nicht Blut geflossen, / Kein Kelch, aus dem sich nicht, in ganz gesunden Leib, / Verzehrend heisses Gift ergossen, / Kein Schmuck, der nicht ein liebenswürdig Weib / Verführt, kein Schwert, das nicht den Bund gebrochen, / Nicht etwa hinterrücks den Gegenmann durchstochen.

A quoi, Méphisto rétorque :

Frau Muhme! Sie versteht mir schlecht die Zeiten. [...] / Verleg' sie sich auf Neuigkeiten! / Nur Neuigkeiten ziehn uns an! $!^{3}$

Il faut remarquer déjà qu'au delà du récit des crimes de Faust, l'évocation du poignard ou du poison "Dolch und Gift" fait discrètement allusion au moins à deux drames bourgeois, Emilia Galotti et Kabale und Liebe. La référence aux années 70 et 80 est littéraire ${ }^{4}$ autant que philosophique et morale. Si l'on ajoute la référence goethéenne aux réflexions que Wilhelm conduit avec Werner et Amélie dans La vocation théâtrale de Wilhelm Meister, on est davantage encore autorisé à situer le dialogue entre Méphisto

2 Faust ... v. 2495 ss. Le texte de Faust sera cité d'après l'édition publiée par Albrecht Schöne: GoETHE, Faust. Text und Kommentare, hrsg. von Albrecht Schöne, Frankfurt/Main, Deutscher Klassiker Verlag, 1999.

3 Ibid., v. 4096-4113.

4 Elle reprend aussi une réflexion de Wilhelm au livre 2 de Wilhelm Meisters Theatralische Sendung: "[...] wie meine Vorgänger mit Dolch und Gift hantiert [....]". Nous y reviendrons. Les œuvres de Goethe autres que Faust sont citées d'après l'édition publiée par Ernst Beutler: GoETHE, Gedenkausgabe der Werke, Briefe und Gespräche, hrsg. von Ernst Beutler, Zürich, Artemis Verlag, 1961. 
et la sorcière en référence à ces deux intertextes et dans le contexte littéraire de l'époque. Wilhelm voulant acquérir une culture théâtrale et écrire, s'interroge sur la mort au théâtre ${ }^{5}$, en particulier chez ses prédécesseurs :

Ich sah, daß meine Vorgänger sich die künstlichste Mühe gegeben hatten, mit Dolch und Gift und andern schädlichen Werkzeugen auf das mannigfaltigste zu hantieren, so daß dem Nachfolger fast keine Kombination mehr übrig blieb.

Cet appel à une modernisation esthétique est évidemment lié à l'historicité des définitions du mal, sur quoi les interventions de Méphisto que l'on a citées veulent attirer l'attention. A une époque que celui-ci estime révolue, le mal est causé et perpétré par des individus alléchés par les brocantes de vieilles sorcières et d'une certaine façon induits en tentation par elles. Le mal serait-il encore imputable, au moins pour une part, à ces puissances néfastes ? Que sont les nouveautés réclamées par Méphisto au nom d'un progrès de la culture ? La scène Auerbachs Keller a montré que les temps modernes ont rendu le mal difficilement définissable. Les jeunes étudiants bringueurs de Leipzig ne reconnaissent ni le vieux diable, ni le hobereau vêtu au goût du jour : "Den Teufel spürt das Völkchen nie", constate Méphisto ! C'est que le mal n'est plus "répertorié" en péchés véniels, mortels, etc., selon un catéchisme tel que celui inculqué à Marguerite. Les instruments du mal moderne ne sont pas à chercher dans les magasins du diable ou des sorcières. La nouveauté, c'est qu'à cette période de seuil l'homme se sait causa sui, ne peut plus assigner à une puissance extérieure la responsabilité ou une part de la responsabilité du mal et croire à une hétéronomie du mal. Dans la scène Trüber Tag. Feld, Méphisto lance à Faust : "Wer war es, der sie ins Verderben stürzte, ich oder du ?", exigeant par là de lui le courage d'une lucidité moderne. Lorsque l'horreur de ce constat s'impose à Faust, celui-ci ne peut que s'écrier : "O wär' ich nie geboren" (Kerker).

Goethe citait ici en même temps la fin d'CEdipe à Colonne et le livre de Job de l'Ancien Testament, la culture de l'Antiquité païenne et celle du JudéoChristianisme. On trouve la même accusation proférée contre des "puissances célestes" chez l'un des personnages les plus tragiques de Goethe, le Harpiste des Années d'apprentissage de Wilhelm Meister.

Wer nie sein Brot mit Tränen aß, [...] / Der kennt euch nicht / Ihr himmlischen
Mächte. / Ihr führt ins Leben uns hinein, / Ihr laßt den Armen schuldig werden, /
Dann überlaßt ihr ihn der Pein, / Denn alle Schuld rächt sich auf Erden

Si la complainte du Harpiste semble incriminer, même de façon floue, une transcendance, que l'homme découvre lorsqu'il fait l'expérience de sa culpabilité, elle relègue cette question de l'origine pour privilégier la vision d'un mal et d'un malheur inhérents à la vie humaine et nécessité de l'Histoire,

5 “[...] die Spekulation einer besonderen Todesart [...]", Wilhelm Meisters Theatralische Sendung, II, 4. Ici p. 610

6 Goethe, Wilhelm Meisters Lehrjahre, in Goethe, Gedenkausgabe, vol. 7, p. 146. 
dont l'homme assume la responsabilité. L'expérience tragique d'un mal sécularisé et intériorisé est celle du Faust de Trüber Tag. Feld et celle du Harpiste, mais rien ne prouve que ce soit totalement et définitivement celle de Goethe.

En effet, l'idée d'un "mal radical" est nuancée par le poète, la représentation augustinienne du péché originel et de la grâce, la conception luthérienne et kantienne d'un "bois tordu" que seule la "grâce divine" peut "sauver", n'est pas totalement la sienne. On connaît le passage de Poésie et Vérité où, regimbant contre le pessimisme radical de la doctrine augustinienne repris alors par certains cercles piétistes qu'il fréquente, Goethe déclare admettre une part de l'héritage luthérien ("die erblichen Mängel"), mais privilégier l'espoir conféré à l'homme par l'autre aspect de la doctrine, la foi en la grâce divine qui pour être agissante suppose l'existence en l'homme d'une aptitude au bien.

\begin{abstract}
Was mich nämlich von der Brüdergemeine sowie von andern werten Christenseelen absonderte, war dasselbige, worüber die Kirche schon mehr als einmal in Spaltung geraten war. Ein Teil behauptete, daß die menschliche Natur durch den Sündenfall dergestalt verdorben sei, daß auch bis in ihren innersten Kern nicht das mindeste Gute an ihr zu finden, deshalb der Mensch auf seine eignen Kräfte durchaus Verzicht zu tun, und alles von der Gnade und ihrer Einwirkung zu erwarten habe. Der andere Teil gab zwar die erblichen Mängel der Menschen sehr gerne zu, wollte aber der Natur inwendig noch einen gewissen Keim zugestehn, welcher, durch göttliche Gnade belebt, zu einem frohen Baume geistiger Glückseligkeit emporwachsen könne. Von dieser letztern Überzeugnung war ich aufs innerste durchdrungen, ohne es selbst zu wissen, obwohl ich mich mit Mund und Feder zu dem Gegenteile bekannt hatte; aber ich dämmerte so hin, das eigentliche Dilemma hatte ich mir nie ausgesprochen ${ }^{7}$.
\end{abstract}

La rédaction de Faust témoigne des interrogations et des doutes du dramaturge. Le Prologue au Ciel introduit une théodicée nouvelle, dans laquelle le salut de l'homme semble décidé de toute éternité et le mal inclus dans le plan divin, comme un aiguillon conduisant in fine au bien et contribuant à une Histoire dont le telos est le Salut. Vision dogmatique et téléologique donc, qui affirme une prédestination au mal et au bien ? Où serait alors la modernité ? Le dernier mot quant à l'interrogation morale est-il celui de Méphisto dans la Nuit de Walpurgis ? A Faust qui espère comprendre dans une ultime révélation ce qu'est "le mal" :

Doch droben möcht' ich lieber sein ! / Schon seh' ich Glut und Wirbelrauch. /

Dort strömt die Menge zu dem Bösen ; / Da muß sich manches Rätsel lösen.

Méphisto, décidément porte-parole de la modernité, réplique : "Da knüpft sich manches Rätsel auch”,

7 Goethe, Dichtung und Wahrheit, III, 15, in GOETHE, Gedenkausgabe, p. 693-694.

8 Faust, v. 4037 ss. 
Afin de sortir du questionnement moral et de moderniser l'imagerie qui y est liée, les sorcières et les diables, le poète moderne inscrit, dès la scène liminaire du drame et de plus en plus une fois qu'il s'est affranchi des scènes obligées dans le cabinet du vieux savant, sa réflexion sur le mal et les figures qui dans la vision médiévale l'incarnent, dans une réflexion plus vaste sur la nature. Si l'affirmation du Prologue d'un salut de Faust et de l'homme malgré son abjection peut être référée à une métaphysique, elle est surtout liée à une "lecture" esthétique et scientifique de la nature élaborée par Goethe. Chronologiquement : dans les années 70, Goethe et les acteurs de la "révolution littéraire" que veut être le Sturm und Drang se réclament d'une nature créatrice, vision que Goethe lecteur de Spinoza conserve et prolonge d'autant plus volontiers qu'elle transfère le pouvoir créateur d'une transcendance à une immanence. Après son installation à Weimar, puis pendant son séjour en Italie et au retour d'Italie, Goethe construit une sorte de philosophie scientifique de la nature. Ce déplacement de la réflexion du domaine moral vers les domaines esthétique et scientifique est au fondement de la modernité de Faust, cette nouvelle optique permet d'intégrer les figures $\mathrm{du}$ folklore ancien que sont diables et sorcières. Comment?

Il faut d'abord reconnaître dans les scènes de sorcellerie et de sabbat, Walpurgisnacht et Hexenküche, l'appartenance des sorcières à la nature, la matière. Dans Walpurgisnacht il est facile de voir comment à partir de perceptions sensibles, sonores puis visuelles, les fantasmes de Faust peuvent sembler prendre corps, devenir des hallucinations et des formes. Dans l'imaginaire populaire, les sorcières sont des créatures mauvaises, qui dévoient les jeunes filles et les jeunes hommes, forniquent avec le diable lors d'orgies sexuelles et criminelles et tuent les enfants. Cette imagerie archaïque est conservée dans Faust, mais le XVIII ${ }^{\mathrm{e}}$ siècle "éclairé", ou en voie de l'être, sait que ce sont les peurs et les jugements d'une société patriarcale dominée par l'Eglise qui créent le personnage de la sorcière, comme incarnation d'une féminité et d'une sexualité dangereuses, à exclure de l'ordre moral et social. La scène Walpurgisnacht retient l'assignation de la sorcière à la nature et à une nature refoulée, et ceci va devenir essentiel dans la dramaturgie moderne.

Faust y entreprend sous la conduite de Méphisto la montée au Brocken; c'est d'abord pour lui une marche dans les forêts, parmi les roches, la végétation et les animaux, le long des ravins, devant les anfractuosités de rochers et les grottes, marche au cours de laquelle le promeneur est assailli de sensations et perceptions multiples. Mais en s'alliant avec le diable, Faust a quitté l'ordre de la raison (“des Denkens Faden ist zerrissen”) et plonge dans un chaos pulsionnel ("Laß uns in den Tiefen der Sinnlichkeit glühende Leidenschaften stillen"), une sorte de chora primitive mythique, il entre dans un univers hallucinatoire, met le pied sur un continent encore inexploré de lui, "die Traum- und Zaubersphäre". Les sorcières y sont omniprésentes, et face à des esprits rationalistes bornés et suffisants qui se vantent d'avoir éradiqué les
\end{abstract}


superstitions par les Lumières, les fantasmagories les plus extravagantes mettent la raison en déroute. Là intervient la modernisation. Se fondant sur ses propres études des sciences de la nature, Goethe revisite la symbolique du Haut et du Bas propre à la théâtralité médiévale et baroque. Dans le Prologue au Ciel, c'est la divinité qui, "d'en haut", définit la place du mal et la position de l'homme dans la création, inscrivant l'une et l'autre dans une perspective théologique et téléologique. A la fin de la scène Kerker, la voix off annonçant le salut de Marguerite est une "voix d'en haut", qui dans le système de l'ordo chrétien est celle d'une transcendance. Le sommet du Brocken, vers lequel montent les sorcières et les diables, est, conformément à la définition du diable comme simia dei, une parodie des hauteurs du Ciel où trônent le Seigneur, les anges et les archanges. Mais, justement, Goethe a abandonné le projet de faire culminer la Nuit de Walpurgis sur un triomphe de Satan, sur une Apocalypse du mal ; on connaît l'avancement de cette composition par les Paralipomènes. Dans un esprit de modernité justement, il a renoncé à un finale qui eût cautionné une vision théologique dualiste.

C'est un autre credo que Goethe expose ici, élaboré parallèlement à la pièce de théâtre par l'observation assidue et la réflexion scientifique. C'est la conviction qu'il existe dans la nature deux forces ou "roues" motrices : "Triebräder". Au fondement de la vie de la nature il y a d'abord une tension antagoniste permanente, si on la considère seulement dans sa matérialité, la nature vit dans des processus incessants de production et destruction, elle est selon les termes de Goethe lui-même une "opération physico-chimique". Il nomme ce jeu d'oppositions la loi de polarité, "Polarität". Mais il n'y a pas de nature, pas de matière, sans esprit. Et si l'on considère la nature dans sa spiritualité, elle apparaît alors comme dotée d'un pouvoir d'élévation, animée d'un formidable élan vers le haut, "Steigerung", qui permet de sortir du cycle infini des morts et naissances et de concevoir un autre niveau d'existence. Les deux principes moteurs, Polarität et Steigerung, sont corollaires. C'est en substance ce que Goethe expose dans une lettre au Chancelier von Müller du 24 mai 1828. Il y revient sur un essai des années 1782-83 du naturaliste Tobler, auquel il a donné son aval, intitulé Die Natur, le fameux Fragment de Tiefurt :

Man sieht [dans le texte de Tobler / Goethe] die Neigung zu einer Art von Pantheismus, indem den Welterscheinungen ein unerforschliches, unbedingtes, humoristisches, sich selbst widersprechendes Wesen zum Grund gedacht ist [...]

Die Erfüllung aber, die ihm fehlt, ist die Anschauung der zwei großen Triebräder aller Natur : der Begriff von Polarität und von Steigerung, jene der Materie, insofern wir sie materiell, diese ihr dagegen, insofern wir sie geistig denken, angehörig ; jene ist in immerwährendem Anziehen und Abstoßen, diese in immerstrebendem Aufsteigen. Weil aber die Materie nie ohne Geist, der Geist nie ohne Materie existiert und wirksam sein kann, so vermag auch die Materie 
sich zu steigern, so wie sich's der Geist nicht nehmen läßt, anzuziehen und abzustoßen ${ }^{9}$.

Le poète plus ou moins panthéiste ("eine Art von"!), qui pose une telle faculté de Steigerung comme moteur de la productivité d'une nature à la fois matière et esprit, est celui qui fait décréter par une transcendance le salut de Faust et de Marguerite. Dans la vision "scientifique", cette accession à un degré plus haut d'existence n'est plus l'intervention d'une transcendance, ou, si elle reste une grâce, alors celle d'un dieu immanent - deus sive natura plutôt une loi de nature, et même le principe de vie de cette nature :

Sie [die Natur] bedient sich hierzu [das Mannigfaltigste hervorzubringen] des Lebensprinzips, welches die Möglichkeit enthält, die einfachsten Anfänge der Erscheinungen durch Steigerung ins Unendliche und Unähnlichste zu vermannigfaltigen $[\ldots]^{10}$.

Deus sive natura! Le Dieu du Prologue se présente comme un jardinier, voit l'homme comme un arbrisseau portant en lui la possibilité de floraison et de fructification ("Blüt' und Frucht"). La partition ancienne du Bien et du Mal et la connotation morale du Haut et du Bas s'effacent au profit de la vision naturaliste d'une croissance organique orientée vers le haut mais n'excluant pas les forces centrifuges aberrantes qui n'obéissent qu'à la loi de polarité et ignorent le principe de Steigerung. On a déjà cité le passage de Poésie et Vérité où la même métaphorique empruntée à la végétation (“[...] Keim, Baum, emporwachen [...]") côtoie la métaphysique ${ }^{11}$.

C'est par l'étude de la botanique et en remettant en question le statisme de la taxinomie au profit d'une vision dynamique, que Goethe s'est dégagé de l'epistémè classique pour entrer dans la science moderne, la science des vivants puis du vivant. L'ouverture à la modernité est dans cette relégation de la métaphysique et la morale au profit de l'étude du vivant, à une période de changement de paradigme où la vie, considérée par Goethe dans ses études scientifiques comme un "phénomène" à explorer, devient objet d'étude ${ }^{12} . \mathrm{Si}$ les sorcières du Brocken restent l'incarnation du mal, c'est que, incluses dans la nature, elles veulent, comme Méphisto, s'opposer à la vie. Les images de la scène Walpurgisnacht font d'elles des tueuses, non seulement la vieille brocanteuse avec ses outils de mort obsolètes, mais les jeunes sorcières qui revendiquent leur stérilité ("wir waschen, wir waschen / Und sind auf ewig unfruchtbar") le sont. Leur retour du Brocken, une fois instauré le royaume de Satan, aurait dû proclamer le triomphe de la mort sur la vie. Mais voilà : Méphisto est contraint d'admettre la vanité de ses efforts de destruction et

9 Die Natur, Fragment [von Christof Tobler], in GoETHE, Naturwissenschaftliche Schriften II, Gedenkausgabe, p. 921 et Erläuterung zu dem aphoristischen Aufsatz Die Natur [Goethe an den Kanzler von Müller], Naturwissenschaftliche Schriften I, Gedenkausgabe, p. 925.

10 Polarität, Essai de 1830, in GOETHE, Naturwissenschaftliche Schriften II, p. 864.

11 Voir note 7.

12 C'est à Michel Foucault que l'on se réfère ici. Voir Michel FouCAult, Les mots et les choses, Paris, Gallimard, 1966, en particulier le chapitre V, Classer. 
l'impossibilité du néant. Qu'il s'agisse d'une créativité immanente infinie, pour qui la mort n'est que "le secret pour avoir beaucoup de vie" (une nature matière) ou d'une puissance de vie éternelle ("der ewig regen, / Der heilsam schaffenden Gewalt") (une nature esprit), il s'agit de cette force néguentropique que Goethe nomme "die Allgegenwart des Lebens". C'est "l'énigme" à laquelle Faust s'affronte, lorsque, fasciné par le pouvoir créateur de la nature, encheiresis naturae, il demande à comprendre comment se maintient la cohésion de l'univers, "was die Welt im Innersten zusammenhält", question à laquelle Méphisto ne peut répondre ${ }^{13}$.

Lorsque Faust, dans son monologue liminaire de la scène Wald und Höhle, prend le temps de contempler la nature, Goethe lui fait formuler l'une de ses propres découvertes scientifiques, majeure :

Du führst die Reihe der Lebendigen / Vor mir vorbei, und lehrst mich meine Brüder / Im stillen Busch, in Luft und Wasser kennen ${ }^{14}$.

Sans excessive audace, on peut lire cette vision "intuitive" (dont se moque Méphisto : "die hohe Intuition"!), ou cet "aperçu" goethéen d'une "chaîne des vivants" et d'une fraternité entre les créatures, comme un écho de la "découverte" par Goethe de l'os intermaxillaire, qui vient confirmer son hypothèse d'une continuité entre les êtres vivants. Le 17 novembre 1784, il écrit à Knebel :

\begin{abstract}
Hier schicke ich dir endlich die Abhandlung aus dem Knochenreiche [...] Ich habe mich enthalten das Resultat, worauf schon Herder in seinen Ideen deutet, schon ietzo merken zu lassen, daß man nämlich den Unterschied des Menschen vom Tier in nichts einzelnem finden könne. Vielmehr ist der Mensch aufs nächste mit den Tieren verwandt. Die Übereinstimmung des Ganzen macht ein jedes Geschöpf zu dem, was es ist, und der Mensch ist Mensch so gut durch die Gestalt und Natur seiner obern Kinnlade, als durch die Gestalt und Natur des letzten Gliedes seiner kleinen Zehe Mensch. Und so ist wieder jede Creatur nur ein Ton eine Schattierung einer großen Harmonie [...].
\end{abstract}

C'est donc bien en nourrissant son drame de sa réflexion sur la nature que le dramaturge peut intégrer et moderniser les sorcières et leur sabbat infernal. Poétiquement, la scène Walpurgisnacht est le prolongement de la scène Wald und Höhle, elle enregistre les implications scientifiques du monologue initial de Faust et les corrections réalistes du diable. Habilement, le poète réunit dans cette perception "matérialiste" de la nature ses convictions scientifiques et l'interprétation ancienne d'une matière "diabolique". Il lui suffit de situer la scène dans l'espace et le temps, par l'indication scénique "Harzgebirg. Gegend von Schierke und Elend" et le titre Walpurgisnacht. En localisant la

13 Il a largement été souligné que Méphisto ne fait que de contribuer au plan divin, mais c'est surtout à la créativité infinie de la nature qu'il contribue, même s'il souhaite la destruction : "Man gehorcht ihren Gesetzen, auch wenn man ihnen widerstrebt ; man wirkt mit ihr, auch wenn man gegen sie wirken will”, GOETHE, Die Natur.

14 Faust, v. 3225 ss. 
scène par ce référent biographique et cette référence mythique, il définit la nature comme affrontement incessant de forces antagonistes qu'il a observé et comme l'ensemble des pulsions ou postulations refoulées par la culture, qu'un imaginaire collectif ancien a ramassé dans les figures des sorcières et autres créatures infernales.

La danse avec la jeune sorcière est un plaisir facile auquel Faust n'a jamais goûté, voire dans cette société une sorte d'interdit (Scène $\mathrm{Am}$ Brunnen : "auf Dorf und Tanzplatz", scène Kerker "wie werden uns wiedersehen, aber nicht beim Tanze"). C'est aussi une image clé de l'essai Die Natur, dont la scène Walpurgisnacht semble une version théâtrale : une sorte de tournis sans fin, dans lequel une nature indifférente parce que considérée dans sa matérialité entraîne les individus jusqu'à ce que mort s'ensuive, le cycle infini ("Kreislauf") des morts et naissances :

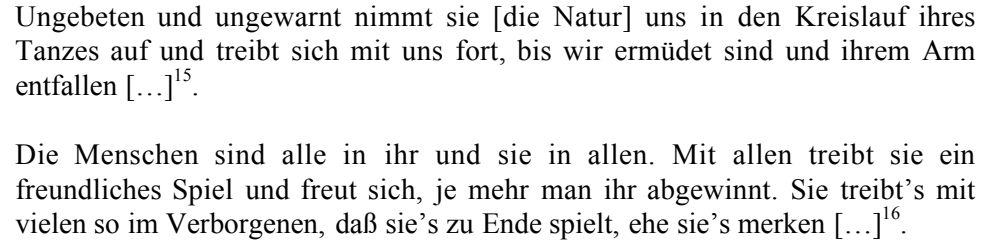

Die Menschen sind alle in ihr und sie in allen. Mit allen treibt sie ein freundliches Spiel und freut sich, je mehr man ihr abgewinnt. Sie treibt's mit vielen so im Verborgenen, daß sie's zu Ende spielt, ehe sie's merken [... $]^{16}$.

Lorsqu'il danse avec la jeune sorcière, Faust est l'un des "enfants" d'une nature amorale, qui ne se soucie pas des individualités qu'elle appelle sans cesse à la vie et qu'elle détruit comme par jeu, à seule fin que la vie continue :

Sie scheint alles auf Individualität angelegt zu haben und macht sich nichts aus den Individuen. Sie baut immer und zerstört immer. [...] Leben ist ihre schönste Erfindung und der Tod ist ihr Kunstgriff, viel Leben zu haben ${ }^{17}$.

Cette danse est donc une danse macabre, elle représente scéniquement le risque d'une acception seulement matérialiste de la nature : s'abîmer dans l'ivresse de cette transe ("daß ich mich nur nicht selbst vergesse") impliquerait une perte de conscience et serait finalement un crime "moderne", un crime contre l'esprit, ou bien contre la nature considérée dans son intégralité, matière et esprit.

Enfin, la modernité des scènes de sorcellerie de Faust réside surtout dans la signification que le lecteur peut leur donner dans le cadre d'une réflexion esthétique, conduite plus volontiers chez Goethe dans l'immanence du texte que dans un discours théorique parallèle. Dans une lettre à Zelter du 4

15 Goethe, GA, vol. 16, p. 921.

16 Ibid., p. 922.

17 Ibid., p. 923 
décembre 1827, Goethe invite son lecteur à prêter attention aux énigmes dont son œuvre abonde, en particulier celles d'ordre esthétique :

Sie [die Deutschen] stolpern über Strohhalmen [...] Ebenso quälen sie sich und mich mit den Weissagungen des Bakis, früher mit dem Hexeneinmaleins und so manchem andern Unsinn, den man dem schlichten Menschenverstande anzueignen gedenkt. Suchten sie doch die psychisch-sittlich-ästhetischen Rätsel, die in meinen Werken mit freigebigen Händen ausgestreut sind, sich anzueignen.

Le sabbat de sorcières de la Nuit de Walpurgis est un appel à une telle lecture. C'est l'infinie productivité d'une nature exclue de l'ordre symbolique, qui est ici théâtralisée. Les premiers essais des années de Sturm und Drang comme les Naturwissenschaftliche Schriften font de la nature une artiste : "Sie ist die einzige Künstlerin" lit-on dans le Fragment de Tiefurt, comme en écho à Werther et à ces essais esthétiques de jeunesse. A partir de ses propres énergies vitales refoulées, donc avec l'aide de Méphisto, Faust crée des images fantasmatiques qui deviennent les figures d'un théâtre onirique et magique ("die Traum- und Zaubersphäre"), ainsi l"'envers de la raison" ou "le mal" contestent un ordre symbolique archaïque. Est ici citée l'esthétique de la création du Sturm und Drang, on entend les appels de Hamann, Herder et du Goethe des années 70 à renouveler l'inspiration et les formes poétiques par une plongée dans la "nature". Le foisonnement d'images de la montée au Blocksberg a la même force contestatrice que les grylles de l'art gothique ou la peinture de Jérôme Bosch, contestataires de l'ordre religieux médiéval et baroque. Explicitement rattachée à la "nature", au soma, cette luxuriance de spectres, de sorcières ou de diables, d'animaux ou de créatures hybrides, de racines térébrantes, suscitée par un imaginaire collectif que l'on a voulu brider, est mise au service d'une esthétique qui cherche à ébranler le pouvoir légiférant du logos, comme les grognements animaux ("der tierische Laut") venaient heurter le discours religieux dans la scène d'exorcisme. Il restera à analyser comment la scène montre les limites de cette esthétique.

Dans les deux scènes Hexenküche et Walpurgisnacht, Goethe conserve aux sorcières et créatures infernales leurs réquisits traditionnels. Il est en effet tout à fait documenté sur les récits ou représentations de sabbats de sorcières et autres synagogae satanae, la précision des paralipomènes qui devaient constituer le finale de la Nuit de Walpurgis en témoigne. Mais le terme "sorcière" apparaît dès la scène Vor dem Tor, où il est une invective désignant une "vieille" aux pratiques douteuses, dangereuse pour la vertu des jeunes filles, entremetteuse de type Marthe, éventuellement "faiseuse d'anges" et à l'avance associée à Méphisto. Elle opère en un lieu mal famé, Burgdorf, opposé au lieu civil qu'est la ville, un locus terribilis qui inspire cette horreur ("mir graut") que Marguerite, marquée par les codes de sa petite bourgeoisie, éprouve spontanément vis à vis de Méphisto, et vis à vis de Faust lorsqu'il est l'acolyte de ce dernier (Kerker), "Heinrich, mir graut vor dir !"). La sorcière est l'un des deux versants de l'image de la femme que Faust porte en lui. 
Cette image est clivée, car surdéterminée par les deux instances qui régissent l'ordre symbolique patriarcal de l'époque, la raison et la religion. Elle est sublime, c'est la beauté d'Hélène et la vertu de Marguerite, et abjecte, c'est la sorcière représentant les pulsions non contrôlées, la nature. On sait que la scène Hexenküche fut écrite en 1788 à Rome, dans les jardins de la Villa Borghese. Les "fantômes nordiques" sont dans une relation de "polarite" avec la culture antique classique : "Schönheit" vs "Barbarei"18 ou encore "Wust von Raserei" vs "himmlisch Bild", "Inbegriff von allen Himmeln". En termes goethéens, cette loi de polarité est active en Faust : si l'on présente à l'œil de la lumière, cet œil suscite l'obscurité, et inversement.

Les didascalies de la scène Hexenküche montrent quelle correspondance étroite existe entre l'antre de la sorcière et le cabinet de travail de Faust au début de la scène Nacht. C'est le même enfermement dans un lieu d'où la vraie vie est absente, la même abondance d'instruments ne servant qu'à "mettre la nature à la torture". Par eux, le lien est établi explicitement entre l'univers des pères ("Urväterhausrat") et celui de la sorcière ("Hexenhausrat"). Le monde patriarcal enfante cette sorcière, qui devient la figure centrale d'un théâtre d'ombres, des fantasmagories d'une lanterne magique agitée par Méphisto devant des fonds de brume ou de fumée ${ }^{19}$ ("Gaukelspiel und Zauberkünste"). La mise en relation d'un théâtre illusionniste avec l'art dramatique, qui, lui, dénonce l'illusion en même temps qu'il la pose, est un appel à une réflexion sur l'illusion au théâtre et dans l'art. A Méphisto dont la volonté de faire le mal n'aboutit jamais et dont le pouvoir est nul, il ne reste que les artifices ("meine Künste").

Les fantasmagories plus ou moins obscènes de Méphisto s'évanouissent lorsque l'œil intérieur de Faust suscite un autre spectacle, également fantasmatique, dont la figure centrale n'a pas le même statut scénique que les sorcières, Lilith ou Baubo. Faust rejette la jeune danseuse dans une même réaction de dégoût que celle ressentie dans l'antre de la sorcière : une souris rouge peu ragoûtante, tirée du bestiaire fantastique des contes et superstitions populaires, s'échappe de la bouche de la jeune danseuse. Le détail sordide sert de transition vers un nouveau spectacle, qui rethéâtralise la thématique du drame bourgeois, la condamnation de la jeune fille séduite devenue mère infanticide ${ }^{20}$. Le drame en miniature que se joue Faust se déroule en trois "actes", séparés par les interventions de Méphisto, et leur enchaînement est un modèle de débat esthétique. Entre le premier et le second "acte" Faust s'implique de plus en plus. A partir d'impressions visuelles encore floues, il accède sinon à l'aveu franc de sa responsabilité dans la tragédie réelle, du moins à la reconnaissance d'avoir été l'amant de Gretchen : "tua res agitur". Le troisième acte est un théâtre de la participation, le spectateur Faust

18 Paralipomène 89 sur Faust : "die Schönheit reißt ihn aus der Barbarei”.

19 Goethe homme de théâtre songeait à une lanterne magique pour représenter la vision de l'Esprit de la terre ou la scène d'exorcisme.

20 Voir Béatrice Dumiche, "Marguerite, un personnage de contestation esthétique", in Béatrice DuMiCHE / Denise BlondeAU (éd.), Faust, modernisation d'un modèle, p. 84. 
parachève le tableau ou la scène en anticipant l'exécution et ajoutant la marque rouge du sang sur le cou. Il restera silencieux jusqu'à son cri de désespoir dans Trüber Tag. Feld. Il est possible, mais non certain, que Faust accède à ce moment à un début de conscience de ses actes. Mais l'intérêt est ailleurs : la construction de la scène par Goethe soulève la question d'une vertu éducatrice, morale du théâtre, d'une scène "moralische Anstalt".

Par cet enchâssement des hallucinations, qui est aussi un procédé habituel chez lui - "wiederholte Spiegelungen" - Goethe interroge la qualité esthétique d'un théâtre qui opère par le pathos, dont les ressorts sont une terreur sans pitié. Il pose une question centrale de la tragédie, celle de la faute tragique et de la rétribution : non pas ou pas seulement au plan moral et social ("die richtende gefühllose Menschheit"), en critiquant l'ordre patriarcal qui pousserait la "fille mère" au désespoir et au meurtre de son enfant; mais au plan esthétique, car la représentation scénique du châtiment opère par la terreur. Il apparaît que ce théâtre de la cruauté est dépassé et peut-être peut-on lire dans sa dénonciation parodique l'autocritique d'un Goethe se souvenant des outrances de son Clavigo. L'hypothèse interprétative est confortée si l'on songe que dans Iphigénie en Tauride Goethe fait fuir les Érinyes, veut mettre un terme aux meurtres, rejette l'expiation ou la rétribution par l'effusion de sang. Au nom du poète Goethe, Thoas interrompt le récit d'Iphigénie : "es sei / Genug der Greuel !"). Le dramaturge substitue à la peine de mort la vertu curative d'une "pure humanité" ("alle menschliche Gebrechen sühnet reine Menschlichkeit"), même si le succès est relatif. La représentation macabre que Faust se donne à lui-même à la fin de la Nuit de Walpurgis reprend les discussions d'esthétique théâtrale de Goethe et Schiller dans les années 90, en particulier leur commun rejet du pathos et de la terreur, encore plus vigoureux chez Goethe qui redoutait de se détruire en écrivant une tragédie.

Avec cette insertion d'un théâtre dans le théâtre, Goethe mobilise les idées qu'il développe dans son interprétation de la poétique d'Aristote ${ }^{21}$, sa discussion de la fameuse catharsis. Il conteste en effet une quelconque "purification" du spectateur par la représentation tragique et envisage en guise de catharsis une résolution du tragique à l'intérieur de la pièce, une issue conciliatrice. Ce débat est poursuivi au début du second Faust par une reprise ironique de la notion, il s'agira d'une catharsis "à la mode des elfes",22 : la fuite et le sommeil de l'oubli.

$\mathrm{Au}$ troisième "acte" du spectacle macabre, Faust spectateur captif s'écrie : "Welch eine Wonne! Welch ein Leiden !". L'absence de sujet dans la formulation laisse ouverte la question de savoir qui jouit et qui souffre. Faust a, une fois engagé avec Méphisto et entré dans la "vraie vie", reconnu les profondeurs ténébreuses de la psyché ("die psychisch-sittlich-ästhetischen 712.

21 Goethe, Nachlese zu Aristoteles' Poetik, in Goethe, Gedenkausgabe, vol. 14, p. 709-

22 Faust : "Erzeigt euch hier nach edler Elfenweise ...Sein Innres reinigt von erlebtem Graus ... / Erst senkt sein Haupt aufs kühle Polster nieder, / Dann badet ihn im Tau aus Lethes Flut ...", Faust, v. 4622 ss. 
Rätsel" de la lettre de Goethe à Zelter), connaissance qui ne s'acquiert que par l'expérience de la vie ... ou / et, sur un mode intellectuel, par la fréquentation des œuvres d'art, en particulier des arts visuels. Il est difficile, lorsque l'on suit le déroulement, comme spectacle dans le spectacle avec parties alternées, de la marche au supplice de la jeune condamnée, de distinguer les positions respectives de Faust et Méphisto. Dans la perspective de la "tragédie" de Faust, Méphisto poursuit sa course et veut traîner Faust "durch das wilde Leben, / durch flache Unbedeutenheit", le faire "frétiller",23 comme une proie prise à l'hameçon. Il doit en conséquence l'empêcher de s'abandonner à l'anamnèse, de tomber dans la paralysie et la sidération. Il veut le diriger vers de nouvelles aventures érotiques ("Ich verbinde dich aufs Neue" ("ich bin der Werber, und du bist der Freier" ) ${ }^{24}$, en lui offrant de la chair fraiche et de nouveaux divertissements, alors que la vision fascinante est celle d'un spectre ou d'une statue froide ("Idol"). Il menace donc Faust de mort, mais d'une mort qui n'est pas celle que lui-même souhaite pour Faust, ni non plus celle que celui-ci imagine.

Dans une autre logique, métapoétique, Méphisto avertit Faust de ne pas confondre leurre et "réalité" ("réalité" n'étant pour cet "esprit menteur" rien d'autre que ses tours de magie !). Se servant de l'ambiguïté du rôle, Goethe fait introduire par le diable une exhortation à ne pas confondre l'art et la réalité. Avec l'image de Méduse, il pose une référence qui symbolise à la fois la décapitation d'une victime monstrueuse et la sidération mortelle du spectateur d'un interdit. Il retient le trouble "étrange" d'un plaisir visuel ambigu (encore une des énigmes "psychisch-sittlich-ästhetisch" de la lettre à Zelter !). On connaît le texte du Voyage en Italie où il se souvient de l'effet produit sur lui par un masque de Méduse vu au Palais Rondanini :

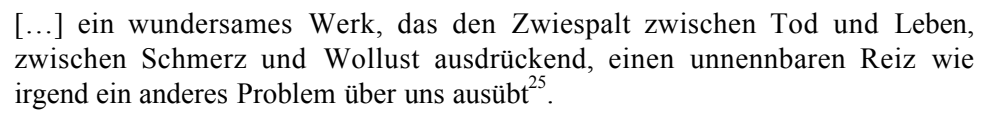

Relever cette dialectique de jouissance et de souffrance, de plaisir et d'angoisse, c'est soulever la question du plaisir trouble pris au spectacle tragique. La fascination ("Ich kann von diesem Blick nicht scheiden") de la mort et de l'horreur est peut-être d'un voyeur, c'est en tout cas l'une des grandes questions d'une esthétique de la réception. Elle est au centre des conversations et échanges épistolaires de Goethe et Schiller sur le sujet. Si Schiller, le théoricien, rédige l'essai Über den Grund des Vergnügens an tragischen Gegenständen, Goethe insère leur réflexion dans sa poésie. Un autre personnage, Wilhelm, l'exprime : se croyant une vocation artistique, il se plonge dans l'histoire et l'esthétique théâtrales. Impressionné par l'existence en l'homme d'une attirance vers le mal ("eine Art Lüsternheit

23 Faust, v.1860 ss.

24 Ibid., v. 4054, v. 4071.

25 GOETHE, Italienische Reise, voir Schöne (n. 2), p. 360-361. 
nach dem Übel ") et d'une propension à jouir de la souffrance ("eine dunkle Sehnsucht nach dem Genusse des Schmerzens"), Wilhelm s'interroge sur le plaisir que peut avoir cet homme à voir souffrir et mourir sur scène :

Man hat oft unter den Kritikern gehandelt, ja wohl gestritten, woher das Gefallen komme, das der Mensch am Drama, besonders am Trauerspiel hat.

Et conclut :

Und selbst grausame zerstörende Begierden [...] haben geheime Wege und Schlupfwinkel, wodurch sie zu den allersüßesten Vergnügungen hinübergehen. Alle diese innerlichen Gänge und Wege werden durch Schauspiele, besonders durch die Tragödie, mit elektrischen Funken durchschüttert, und ein Reiz ergreift den Menschen; je dunkler er ist, je größer wird das Vergnügen ${ }^{26}$.

En faisant ainsi des sorcières et du diable les acteurs d'un mauvais théâtre, illusionniste et cruel, Goethe transforme un folklore archaïque en une avancée vers la modernité théâtrale. Il propose de poursuivre cette réflexion esthétique en faisant se heurter, dans le Walpurgisnachtstraum, une pièce de dilettante, jouée par des dilettantes, et le théâtre de Shakespeare. Mais il aura alors épuisé l'apport esthétique des sorcières.

26 GOETHE, Wilhelm Meisters Theatralische Sendung, livre II, chapitre 4, p. 610, puis II, 5, p. 615,618 